\title{
NEW LABORATORY TESTS FOR DIAGNOSIS AND PROGNOSIS OF BREAST CANCER
}

Guilherme N. Corrêa1, Mateus F. O. Vilela1, Bráulio B. Rodrigues¹, Diogo T. de Lima¹, Gabriela F. Araújo', Fábio F. Marques ${ }^{1}$, Mirian P. Silva ${ }^{1}$

${ }^{1}$ Centro Universitário de Anápolis (UniEVANGÉLICA) - Anápolis (GO), Brazil.

Objectives: To identify new laboratory tests for prognosis and diagnosis of breast cancer. Methodology: Literature review, with search of articles in the databases PubMed and Medline, using the descriptors: new laboratory tests, breast cancer, prognosis, diagnosis. A total of 75 articles were selected, of which 41 were adequate for the review research. The inclusion criteria were more recent articles and of high theoretical value for this review. Results: The laboratory tests identified can be divided into three groups: the first is those that use computer engineering to form images, three-dimensional models of the breast and to track tumor cells. In the second are those that investigate gene and epigenic expressions. In the third group we have histochemical tests of specific substances associated with breast cancer. In the first group, there are several applications of computer technology, such as CellSearch System software and EPISPOT, which use specialized microscopy to identify tumor cells in breast tissue biopsies. Another example of joint work between pathology and computation are high-resolution imaging exams such as the US-CNBs, a highly-defined ultrasound examination, the ED-CSCT, an examination that uses X-ray scatter to calculate the type and the size of microcalcifications that can occur in breast cancer. Computer technology also enabled the creation of a three-dimensional model of the breast, enabling the physician to accurately visualize the tumor in the midst of tissue layers. In the second group, gene expression screening focuses on joint monitoring, observing the traditional genes, such as BRCA1/BRCA2 and Ki67, and other associated ones, such as the CA72-4 carcinogen antigen and the GRP94 and FN14 biomarkers, since their expression is directly associated with breast cancer. In addition to the genetic markers, epigenetic studies revealed a correlation between methylation of the RASSF1A and CDH1 gene promoters and the prognosis of metastasis in breast cancer. In the third group are improved histochemical tests, which guarantee greater accuracy and specificity, such as the use of chromogenic and fluorescent methods in the evaluation of the estrogen receptor, the automated use of the FISH protocol to reduce tumor cell damage, avoiding sample loss, the analysis of the organizing regions of the nucleus of a tumor cell, the change in the technique of histochemical staining for a better visualization of the endothelial growth factors 2 . It is also worth noting the elaboration of new protocols for exams, such as for the analysis of the factor gene of endothelial growth 2. Conclusion: The evolution of technology allowed not only the creation of more specific exams but also the improvement of old techniques. In this way, there are now tools that help the physician in the accurate diagnosis and prognosis of breast cancer, allowing a more specific visualization of the health-disease process, that is, the tests allow to identify the uniqueness of the pathology that affects the individual, either by more accurate imaging tests, either by enhancement and identification of new biomarkers, or by enhancement of old histochemistry techniques. These are factors that allow identifying the characteristics of the pathology that affects the individual, increasing the possibilities of diagnosis, treatment and prognosis by the physician. 